International Mathematical Forum, 2, 2007, no. 6, 285 - 295

\title{
A Study on F-Algebras and Almost F-Algebras
}

\author{
Mehmet Şahin \\ University of Gaziantep \\ Faculty of Arts and Sciences, Department of Mathematics \\ Gaziantep, Turkey \\ mesahin@gantep.edu.tr
}

\begin{abstract}
In this paper the properties of almost f-algebras and f-algebras given by [1], [2], [3], [4] have been studied. The most important one among these properties is that almost $\mathrm{f}$-algebras and archimedian almost $\mathrm{f}$-algebras are commutative. Furthermore nilpotent elements of f-algebras and Archimedian f-algebras have been studied.
\end{abstract}

Mathematics Subject Classification: 06F20

Keywords: f-Algebras, Archimedean f-Algebras, linear space

\section{INTRODUCTION}

Let $\mathrm{A}$ is a Riesz space and simultaneous algebras. For every $0 \leq a, b \in A$ if $0 \leq a \cdot b, \mathrm{~A}$ is called Riesz algebra. An A Riesz algebra providing following characteristic, " $a \cdot b \in A$ and for every $c \geq a, a \wedge b=0 \Rightarrow a c \wedge b=c a \wedge b=0 "$ is called $\mathrm{f}$-algebra. Let $\mathrm{X}$ be an topological space, linear space $\mathrm{c}(\mathrm{X})$ of real valued continuos functions, defined on $\mathrm{X}$ is a typical example of $\mathrm{f}$-algebras. The definition of f-algebras has been firstly defined in the article called "lattice ordered rings. An. Acad. Brasil. C:28 (1956), (41-49) by G.Birkhoff and R.S.Pierce [5]".

In their article Birkhoff and Pierce showed that f-algebra A provides the property of $n|a b-b a| \leq a^{2}+b^{2}$ for every $a \cdot b \in A$ and $n \in N$. This indicates that Archimedean f-algebras are commutative. C.B.Huijmans and B.De Pagter also showed that Archimedean f-algebras are commutative using different method in their article called "Ideal-theory in f-algebras. Trans. Amer. Math. Soc. 269, 225-245". [6]. 
A Riesz algebra $\mathrm{A}$ is called almost f-algebras if it provides $a \wedge b=0 \Rightarrow a \cdot b=0$ for every $a \cdot b \in A$. But [3] have stated that the reverse is not true.

In their article these authors studied the commutative property of Archimedean f-algebras.

Definition 1. Let $E$ be an ordered vector space. If the order of $\leq$ provides $y \leq 0$ when $n x \leq y$ for each $n \in N, E$ is called Archimedian.

Definition 2. i: As [3] suggested, L is a vector space and partically ordered, if for each $x, y \in L$

1. For each $z \in L, x+z \leq y+z$ while $x \leq y$

2. For $0 \leq \alpha \in R, \alpha x \geq 0$ while $x \geq 0$ are provided, $L$ is called partially ordered reel vector spaces

ii: $L$ is partially ordered reel vector space if for each $x, y \in L, x \vee y=$ $\sup (x, y)$ and $x \wedge y=\inf (x, y)$ exists on $L, L$ is called Riesz space (vector lattice).

iii: Let $L$ is an Riesz space and $L$ is a subspace of $K$. If for each $x, y \in K$, $x \vee y$ and $x \wedge y$ are the elements of $K, K$ is called Riesz subspace of $L$.

iv: Let $L$ is an Riesz space and $J$ is a subspace of $L$. If $x \in L, y \in J$ and $0 \leq|x| \leq|y|, J$ is called ordered ideal in $L$.

$\mathbf{v}$ : Let $B$ is an ordered ideal of $L$. If any subset of $B$ has a supremum in $L$ and it also bounds to, $B$ is called a bound in $L$

i: The minimum ordered ideal including $\mathrm{x}$ element of $\mathrm{L}$ is called ordered ideal produced by $\mathrm{x}$ and it is showed indicated $I_{x}$

$$
I_{x}=\{y \in L:|y| \leq|\lambda x|, \lambda \in R\}
$$

ii: It is obviously that each ordered ideal is a Riesz subspace. The smallest band including $\mathrm{x}$ element is called band produced by $\mathrm{x}$. For $x, y \in L$ if $|x| \wedge|y|=0$, $\mathbf{x}$ and $\mathrm{y}$ elements are perpendicular and it is shown by $x \perp y$.

iii: For $D \subset L$, the perpendicular of $\mathrm{D}$ is

$$
D^{d}=\{x \in L: x \perp y \text { for } y \in D\}
$$

for each $D \subset L, D^{d}$ is a band in Archimedean Riesz space. $D^{d d}$ is showed $\left(D^{d}\right)^{d}$. If upper bounded set which is not empty has supremum in $\mathrm{L}, \mathrm{L}$ is called exact Dedekind.

The set of $\{x: x \in E$ and $x \geq 0\}$ showed with $E_{+}$is called positive cone of $\mathrm{E}$ and it is elements are positive. Let $\mathrm{L}$ is Riesz space for each $x \in L$, the 
notations of

$$
x^{+}=\sup (x, 0), x^{-}=\inf (x, 0) \text { and } x \vee y=\sup (x, y), x \wedge y=\inf (x, y)
$$

can be used.

\subsection{Ordered Bounded Operators.}

Definition 3. Let $T$ be an linear transformation, for each $D \subset L$ ordered bounded set, if $T(D) B$ ordered bounded in M.T is called ordered bounded operator. All the sets (of ordered bounded operators) from $L$ to $M$ is showed $L_{b}(L, M)$ if for $T_{1}, T_{2} \in L_{b}, T_{1} \leq T_{2} \Leftrightarrow$ for each $x \in L_{T}, T_{1} . x \leq T_{2} . x, L_{b}(L, M)$ is a ordered vector space. Let $T$ be an operator from $L$ to $M$. If for each $x \in L_{T} T_{x} \geq 0$. $T$ is called positive operator. Each positive operator is ordered bounded. Therefore the difference between two operators is ordered bounded. The positive part of $L_{b}(L, M)$ consists of positive operators. If $M$ is an exact Dedekind, $L_{b}(L, M)$ consists of Dedekind operators. If $M$ is exact Dedekind, $L_{b}(L, M)$ exact Dedekind is Riesz space. In this case every ordered bounded can be written the difference of two positive operators.

Definition 4. While $T \in L_{b}(L, M)$ is an. We show the set of ordered continuous operators $L_{n}(L, M) . L_{n}(L, M)$ is the subspace of $L_{b}(L, M)$.If $M$ is an exact Dedekind $L_{n}(L, M)$ is a band. If $M=R, L_{0}(L, R)=L^{-}$is showed $L_{n}(L, R)=L_{n}^{-}$. $L^{-}$is called ordered dual of $L, L_{n}^{-}$is called ordered continuous dual of $L$.

Definition 5. Let $T$ is an operator from $L$ to $M$. If for each $x, y \in L, x \wedge y=$ $0, T(x) \wedge T(y)=0, T$ is called Riesz homomorphism (lattice homomorphism). Each Riesz homomorphism is positive. The necessary and sufficient condition for $T$ being Riesz homomorphism of transformation from $L$ to $M$ is for $x, y \in$ $L, T(x \wedge y)=T(x) \wedge T(y)$ or $T(x \vee y)=T(x) \vee T(y)$ or each $x \in L, T\left(x^{+}\right)=$ $(T x)^{+}$. Each kernel of Riesz homomorphism is ideal. If it is ordered continuous zero space is a band. If any for $f \in L^{-}$,

$$
N_{f}=\{x \in L: \text { If }(|x|)=0\}
$$

is an ideal. In $f \in L_{n}^{-}$, it is a band. If $f$ is Riesz homomorphism Krnf $=N_{f}$

\subsection{Orthomorphism and Its Characteristics.}

Definition 6. Let $\pi$ be operator from $L$ to $L$. For each $B$ band of $L$, if $\pi(B) \subset$ $B, \pi$ is called an operator which makes the bands constant.

Lemma 1. See [12] For $\pi: L \rightarrow L$ operator followings are equivalent

i: $\pi$ makes the band constant 
ii: For $\forall D \subset L$, where $D \neq 0 ; \pi\left(D^{d}\right) \subset D^{d}$

iii: If $x, y \in L, x \wedge y=0 \Rightarrow \pi \cdot x \perp y$

iv: For $x, y \in L$, if $x \perp y \Rightarrow \pi \cdot x \perp y=0$

v: For each $x \in L, \pi \cdot x \in\{x\}^{d d}$

Definition 7. An ordered bounded transformation which makes bands of $L$ constant is called orthomorphism on L. The sets of orthomorphism is written by $\operatorname{Orth}(L)$

Theorem 2. Let I be the unit of Dedekind $\operatorname{Orth}(L), x, y \in L, 0 \leq x \leq y$ there is $\pi \in \operatorname{Orth}(L), \pi x=y$ and $0 \leq \pi \leq I$. (see [12])The characteristics of orthomorphism can be stated followings.

i: Each orthomorphism of $L$ is ordered continuous

ii: For every $U \in L_{+}, \pi_{1}, \pi_{2} \in \operatorname{Orth}(L),\left(\pi_{1} \wedge \pi_{2}\right)(U)=\pi_{1}(U) \wedge \pi_{2}(U)$ and $\left(\pi_{1} \vee \pi_{2}\right)(U)=\pi_{1}(U) \vee \pi_{2}(U)$

iii: For $\pi \in \operatorname{Orth}(L), N_{\pi}=\{x \in L:\{|x|(|x|=0)\}$ zero space is a band.

iv: Let $\pi_{1}, \pi_{2} \in \operatorname{Orth}(L)$ and $D \subset L$. Every $x \in D$ if $\pi_{1}(x)=\pi_{2}(x)$ for every $y \in D^{d d}, \pi_{1}(y)=\pi_{2}(y)$. As a result, if $L$ has a weak ordered unit $\left(e^{d d}=L\right)$, two orthomorphism can be considered equal if they are equal on $e$.

v: If $\pi \in \operatorname{Orth}(L), R_{\pi}$ is the image set of $\pi, N_{\pi}=R \frac{d}{\pi}$

vi: Let $\pi \in \operatorname{Orth}(L)$ and $\pi^{2}=\pi, R_{\pi}$ is a band projection and $\pi$ is a band projection on $R_{\pi}$. The proof of this theorem can be seen in [10] and [12].

Definition 8. The set of

$$
Z(L)=\left\{\pi \in L_{b}(L):-\lambda I \leq \pi \leq \lambda I, \lambda \in R_{T}\right\}
$$

is called center of $L$. If $Z(L) \subset \operatorname{Orth}(L)$ and $L$ is a Dedekind, the ideal produced by the unit $L_{b}(L)$ is $Z(L)$. If $L$ Dedekind is exact, $Z(L)$ and $\operatorname{Orth}(L)$ are Dedekind Riesz spaces. If $Z(L)$ is a norm of space and Banach lattice, $\operatorname{Orth}(L)=Z(L)$ ( see [12] ), when $L$ is regular then $Z(L)$ is a Banach space.

\subsection{F-Algebras and Properties.}

Definition 9. Let $X$ is a vector space. The function called multiplication function from $X \times X$ to $X$ for every $x, y, z \in X$ and $\alpha \in R$, provides following properties;

i: $x(y z)=(x y) z$

ii: $x(y+z)=x y+x z$ and $(x+y) z=x z+y z$ 
iii: $\alpha(x y)=(\alpha x) y=x(\alpha y)$

$X$ is an associative algebra.

Definition 10. Let $A$ be a Riesz space and associative algebra. If for every $a, b \in A_{+}, a \cdot b \in A_{+}, A$ is called Riesz algebra. Let $A$ be Riesz algebra, when $a, b \in A, a \wedge b=0$ if for every $c \in A_{+} ; a \cdot c \wedge b=c \cdot a \wedge b=0, A$ is called f-algebra.

Definition 11. Let $A$ is an f-algebra and $a \in A$. If for at least one $k$ natural number, while $a^{k}=0, a=0, A$ is called half-prime f-algebra. The necessary and sufficient condition to make $A$, -algebra semi-prime when $a^{2}=0, a=0$.

Theorem 3. If $A$ is an f-algebra

i: For $a, b \in A$ and $c \in A_{+}$

$c \cdot(a \wedge b)=c \cdot a \wedge c \cdot b,(a \wedge b) \cdot c=a \cdot c \wedge b \cdot c$ and $c \cdot(a \vee b)=c \cdot a \vee c \cdot b,(a \vee b) \cdot c=a \cdot c \vee b \cdot c$

ii: For $a, b \in A$, if $a \perp b \Rightarrow a \cdot b=0$

iii: If $A$ is called semi-prime and $a, b \in A, a \perp b \Leftrightarrow a \cdot b=0$

iv: If $A$ is with unit, it is semi-prime.

v: If $A$ is Archimedean, it is commutative.

The proof of this preposition can be seen in [12].

\section{Almost F-Algebras}

2.1. Every Archimedean F-Algebras is Commutative. Almost f-algebras for $\forall a, b \in A$, the difference between $a b$ and $b a$ is shown as [3];

$$
[a, b]=-[b, a]=a b-b a
$$

for every $a, b \in A$, since

$$
\begin{aligned}
(a-a \wedge b) \wedge(b-a \wedge b) & =(b-a \wedge b) \wedge(a-a \wedge b)=0 \\
(a-a \wedge b) \cdot(b-a \wedge b) & =(b-a \wedge b) \cdot(a-a \wedge b)=0
\end{aligned}
$$

is obtained. Furthermore,

$$
\begin{aligned}
a b & =a(a \wedge b)+(a \wedge b) b-(a \wedge b)^{2} \\
& =(a-a \wedge b)(a \wedge b)+(a \wedge b)(b-a \wedge b)+(a \wedge b)^{2} \\
b a & =b(a \wedge b)+(a \wedge b) a-(a \wedge b)^{2} \\
& =(b-a \wedge b)(a \wedge b)+(a \wedge b)(a-a \wedge b)+(a \wedge b)^{2}
\end{aligned}
$$


Then

$$
\begin{aligned}
{[a, b] } & =[a-a \wedge b, a \wedge b]+[a \wedge b, b-a \wedge b] \\
& =-[a \wedge b, a-a \wedge b]+[a \wedge b, b-a \wedge b]
\end{aligned}
$$

are obtained.

Lemma 4. If $a, b \in A^{+}$and $\theta>0$

$0 \leq(a-a \wedge b)(a \wedge b)-(a-a \wedge(1+\theta) b)\left(a \wedge b \wedge \theta^{-1}(a-a \wedge b)\right) \leq \theta b^{2}$ and $0 \leq$ $(a \wedge b)(a-a \wedge b)-\left(a-b \wedge \theta^{-1}(a-a \wedge b)\right)(a-a \wedge(1+\theta) b) \leq \theta b^{2}$

Proof. $a \wedge(1+\theta) b \leq a \wedge b+a \wedge \theta \cdot b$ is clear. Then $0 \leq a \wedge(1+\theta) b-a \wedge b \leq \theta \cdot \mathrm{b}$ is obtained. If the right part is multiplied by $a \wedge b$

$$
0 \leq(a \wedge(1+\theta))(a \wedge b)-(a \wedge b)^{2} \leq \theta \cdot b(a \wedge b) \leq \theta \cdot b^{2}
$$

is obtained. Writing once again

$$
0 \leq(a-a \wedge b)(a \wedge b)-(a-a \wedge(1+\theta) b)(a \wedge b) \leq \theta b^{2}
$$

is obtained. Then

$$
\begin{aligned}
0 & \leq a \wedge b-a \wedge b \wedge \theta^{-1}(a-a \wedge b) \\
& =\theta^{-1}(\theta(a \wedge b)-(\theta(a \wedge b) \wedge(a-a \wedge b)) \\
& =\theta^{-1}(\theta(a \wedge b)-((\theta+1)(a \wedge b) \wedge a-(a \wedge b))) \\
& =\theta^{-1}((\theta+1)(a \wedge b)-(\theta+1) a \wedge(\theta+1) b \wedge a) \\
& =\theta^{-1}((\theta+1)(a \wedge b)-a \wedge(\theta+1) b) \leq \theta^{-1}((\theta+1) b-a \wedge(\theta+1) b)
\end{aligned}
$$

Then $(a-a \wedge(\theta+1) b) \wedge((\theta+1) b-a \wedge(\theta+1) b)=0$ can be seen easily. Then followings are obtained;

$a \wedge b-a \wedge b \wedge \theta^{-1}(a-a \wedge b) \perp(a-a \wedge(\theta+1) b)$ and $(a-a \wedge(\theta+1) b) \cdot(a \wedge b-$ $\left.a \wedge b \wedge \theta^{-1}(a-a \wedge b)\right)=0$ and

$$
(a-a \wedge(\theta+1) b) \cdot(a \wedge b)=(a-a \wedge(\theta+1) b) \cdot\left(a \wedge b \wedge \theta^{-1}(a-a \wedge b)\right)
$$

is obtained. First inequality is obtained through 2.3 and 2.2. the second inequality can be obtained in the same way [3].

Lemma 5. For every $a, b \in A^{+}$and $\theta>0$,

$$
\left|[a, b]+\left[f_{0}(a, b, \theta), f_{1}(a, b, \theta)\right]-\left[f_{0}(b, a, \theta), f_{1}(b, a, \theta)\right]\right| \leq 2 \theta\left(a^{2}+b^{2}\right)
$$


Proof. In 2.1 Using triangle inequality $[a, b]=-[a \wedge b, a-a \wedge b]+[a \wedge b, b-a \wedge b]$ the following is obtained:

$$
\begin{gathered}
\quad\left|[a \wedge b, a-a \wedge b]-\left[f_{0}(a, b, \theta), f_{1}(a, b, \theta)\right]\right| \\
=\left|(a \wedge b)(a-a \wedge b)-(a-a \wedge b)(a \wedge b)-\left(f_{0}(a, b, \theta), f_{1}(a, b, \theta)-f_{1}(a, b, \theta), f_{0}(a, b, \theta)\right)\right| \\
=\left|((a \wedge b)(a-a \wedge b))-\left(f_{0}(a, b, \theta) f_{1}(a, b, \theta)+(a-a \wedge b)(a \wedge b)-f_{1}(a, b, \theta) f_{0}(a, b, \theta)\right)\right| \\
\leq\left|((a \wedge b)(a-a \wedge b))-\left(f_{0}(a, b, \theta) f_{1}(a, b, \theta)\right)\right|+\left|(a-a \wedge b)(a \wedge b)-f_{1}(a, b, \theta) f_{0}(a, b, \theta)\right| \\
\leq 2 \cdot \theta \cdot b^{2}
\end{gathered}
$$

Beside, if $a$ and $b$ interchangeable

$$
\left|[a \wedge b] b-a \wedge b\left[f_{0}(b, a, \theta), f_{1}(b, a, \theta)\right]\right|
$$

is also obtained. Applying 2.1 and triangle inequality once more, the wanted inequality is obtained. For every n natural numbers, $D_{n}$ shows all sets of sequences which consist of zeros and ones and whose length is $n$, that is for

$$
D_{n}=\left\{\varepsilon=\left(\varepsilon_{1}, \varepsilon_{2}, \ldots, \varepsilon_{n}\right), \forall 1 \leq i \leq n, \varepsilon_{1}=0 \text { or } \varepsilon_{1}=1\right\} .
$$

If $\left(\varepsilon=\varepsilon_{1}, \varepsilon_{2}, \ldots, \varepsilon_{n}\right) \in D_{n}$ for $\left(\varepsilon_{1}, \varepsilon_{2}, \ldots, \varepsilon_{n}, 0\right) \in D_{n+1}$ can be written [3]. It also valued for $(\varepsilon, 1)$. Furthermore, we write $|\varepsilon|=\sum_{r=1}^{n} \varepsilon$

Definition 12. Let $a, b, \alpha, \beta \in A^{+}$and $\tau=\left(\tau_{1}, \tau_{2}, \ldots\right)$ be the sequence of increasing positive reel numbers. For every given $k=1,2, \ldots$ and $\varepsilon \in D_{k}$, with induction method. Let's define $\alpha(\varepsilon)=\alpha(\tau, a, b, \varepsilon)$ elements of $A^{+}$, when $\alpha(0)=a, \alpha(1)=b$ for

$$
\varepsilon=\left(\varepsilon_{1}, \varepsilon_{2}, \ldots, \varepsilon_{k}\right) \in D_{k}
$$

and $i=0,1$ as

$$
\alpha((\varepsilon, 1))=f_{i}\left(\alpha(\varepsilon), \alpha\left(\varepsilon_{1}, \varepsilon_{2}, \ldots, \varepsilon_{k-1}, 1-\varepsilon_{k}\right), \tau_{k}\right)
$$

In this definition [3]

$$
\begin{aligned}
\alpha((1,0)) & =f_{0}\left(\alpha(1), \alpha(0), \tau_{1}\right)=a \wedge b \wedge \tau_{1}^{-1}(b-a \wedge b) \\
\alpha((0,0,0,1)) & =f_{1}\left(\alpha(0,0,0), \alpha(0,0,1), \tau_{3}\right) \\
& =\alpha((0,0,0))-\alpha((0,0,0)) \wedge\left(1+\tau_{3}\right) \alpha(0,0,1)
\end{aligned}
$$

Lemma 6. i: For $\varepsilon=\left(\varepsilon_{1}, \varepsilon_{2}, \ldots, \varepsilon_{k}\right) \in D_{k}$ and $1 \leq r \leq k, \alpha\left(\left(\varepsilon_{1}, \varepsilon_{2}, \ldots, \varepsilon_{k}\right)\right) \leq$ $\alpha\left(\left(\varepsilon_{1}, \ldots, \varepsilon_{\tau}\right)\right)$,

ii: For $\varepsilon, \varepsilon^{\prime} \in D_{k}, \varepsilon \neq \varepsilon^{\prime}$ and $i, j \in\{0,1\}, \alpha((\varepsilon, i)) \wedge \alpha((\varepsilon, j))=0$

iii: For $\varepsilon \in D_{k}, \alpha((\varepsilon, 0)) \leq(1 / k+1)(\alpha(0)+\alpha(1))$ and $\alpha((\varepsilon, 0)) \leq \alpha(0)+$ $\alpha(1)$

Proof. $\quad$ i: Using $0 \leq f_{0}(a, b, \theta)+f_{1}(a, b, \theta) \leq \alpha$ we can see $f_{0}(a, b, \theta) \leq$ $a_{1} f_{1}(a, b, \theta) \leq a$ 
ii: Using (i) for every $\varepsilon \in D_{k}, k=(0,1,2, \ldots), \varepsilon\left(\left(\varepsilon^{\prime}, 0, i\right)\right) \wedge \alpha((\varepsilon, 1, i))=0$ for $\mathrm{k}=0$ if $\varepsilon \notin 0$.

iii:

$$
\begin{aligned}
\alpha((0,0)) & =f_{0}\left(\alpha(0), \alpha(1), \tau_{1}\right) \leq \tau_{1}^{-1}(\alpha(0)-\alpha(0) \wedge \alpha(1)) \\
& =\tau_{1}^{-1}(\alpha(0)-\alpha(1))^{+} \in\left\{(\alpha(0)-\alpha(1))^{+}\right\}^{d d}
\end{aligned}
$$

and

$$
\begin{aligned}
\alpha((0,1)) & =f_{1}\left(\alpha(0), \alpha(1), \tau_{1}\right)=\alpha(0)-\alpha(0) \wedge\left(1+\tau_{1}\right) \alpha(1) \\
& \leq(\alpha(0)-\alpha(1))^{+} \in\left\{(\alpha(0) \alpha(1))^{+}\right\}^{d d}
\end{aligned}
$$

Similarly

$$
\alpha((i, j)) \in\left\{(\alpha(1)-\alpha(0))^{+}\right\}^{d d},(j=1,2)
$$

So for

$$
\forall i, j \in\{a, 1\}, \alpha((0, i)) \wedge \alpha((1, j))=0 .
$$

Then (ii) is obtained.

iv: Induction will be used for the first inequality For $k=0$,

$$
\alpha(0), \alpha(1) \leq \alpha(0)+\alpha(1)
$$

For $k=1$,

$$
\alpha((0,0)), \alpha((1,0)) \leq \alpha(0) \wedge \alpha(1) \leq \frac{1}{2}(\alpha(0)+\alpha(1))
$$

Let suppose the inequality is right for $\mathrm{k}$. Let

$$
\begin{aligned}
\varepsilon & =\left(\varepsilon_{1}, \ldots, \varepsilon_{k}, \varepsilon_{k+1}\right) \in D_{k+1} \\
\varepsilon^{*} & =\left(\varepsilon_{2}, \ldots, \varepsilon_{k}, \varepsilon_{k+1}\right), \varepsilon=\left(\varepsilon_{1}, \varepsilon^{*}\right)
\end{aligned}
$$

and $\tau^{*}=\left(\tau_{2}, \ldots\right)$ from induction and Definition 12, from

$$
\begin{aligned}
& 0 \leq f_{0}(a, b, \theta)+f_{1}(a, b, \theta) \leq \alpha \\
& \alpha((\varepsilon, 0))=\alpha\left(\tau^{*}, \alpha\left(\left(\varepsilon_{1}, \varepsilon_{2}\right)\right), \alpha\left(\varepsilon_{1}, 1-\varepsilon_{2}\right)\right) \\
&\left(\varepsilon^{*}, 0\right) \leq\left(\frac{1}{k+1}\right)\left(\alpha\left(\left(\varepsilon_{1}, \varepsilon_{2}\right)\right)+\alpha\left(\left(\varepsilon_{1}, 1-\varepsilon_{2}\right)\right)\right) \leq \frac{1}{k+1} \cdot \alpha\left(\varepsilon_{1}\right)
\end{aligned}
$$

Besides from the definition of $\alpha(\varepsilon), \alpha\left(\left(\varepsilon_{1}, \ldots, \varepsilon_{k}, \theta\right)\right)$. Repeating this, the following inequality is obtained.

$$
\alpha((\varepsilon, 0)) \leq \alpha((\varepsilon, 0)) \leq\left(1-\varepsilon_{1}\right)
$$


second side of the inequality is obtained from

$$
0 \leq f_{0}(a, b, \theta)+f_{1}(a, b, \theta) \leq a
$$

From 2.4 and 2.5

$$
(k+2) \alpha((\varepsilon, 0)) \leq \alpha\left(\varepsilon_{1}\right)+\alpha\left(1-\varepsilon_{1}\right)=\alpha(0)+\alpha(1)
$$

is obtained and the inequality of (iii) is also proved. For second equality, (i) and $\alpha((\varepsilon, 1)) \leq \alpha\left(\varepsilon_{1}\right)$ and $\alpha((\varepsilon, 1)) \leq \alpha\left(\varepsilon_{1}\right) \leq \alpha(0)+\alpha(1)$ are observed and then proof is completed [3].

Definition 13. For $k=1,2 \ldots$

$$
c_{k}=\sum_{\varepsilon \in D_{k}}(-1)^{|\varepsilon|+k}[\alpha((\varepsilon, 0)), \alpha((\varepsilon, 1))]
$$

For example

$$
\begin{gathered}
c_{1}=\sum_{\varepsilon \in D_{1}}(-1)^{|\varepsilon|+1}[\alpha((\varepsilon, 0)), \alpha((\varepsilon, 1))]=-[\alpha((0,0)), \alpha((0,1))]+[\alpha((0,1)), \alpha((1,1))]= \\
-\left[f_{0}\left(a, b, \tau_{1}\right), f_{1}\left(a, b, \tau_{1}\right)\right]+\left[f_{0}\left(b, a, \tau_{1}\right), f_{1}\left(b, a, \tau_{1}\right)\right]
\end{gathered}
$$

from Lemma 5

$$
\left|[\alpha, b]-c_{1}\right| \leq 2 \cdot \tau_{1}\left(a^{2}+b^{2}\right) \text { then } c_{0}=[a, b] \text { so }\left|c_{0}-c_{1}\right| \leq 2 \cdot \tau_{1}\left(a^{2}+b^{2}\right)
$$

is obtained [3].

\section{Lemma 7.}

$$
\left|c_{k}-c_{k+1}\right| \leq 2 \cdot \tau_{k+1}\left(a^{2}+b^{2}\right),(k=0,1,2, \ldots)
$$

Proof. Inequality is obtained for $k=0$ in Definition 13 so let $k \geq 1$,

$$
\begin{gathered}
c_{k+1}=\sum_{\varepsilon \in D_{k+1}}(-1)^{|\varepsilon|+k+1}[\alpha((\varepsilon, 0)), \alpha((\varepsilon, 1))]=\sum_{\varepsilon \in D_{k}}(-1)^{|\varepsilon|+k}[\alpha((\varepsilon, 0,0)), \alpha((\varepsilon, 0,1))]+ \\
\sum_{\varepsilon \in D_{k}}(-1)^{|\varepsilon|+k}[\alpha((\varepsilon, 1,0)), \alpha((\varepsilon, 1,1))] \\
c_{k}-c_{k+1}= \\
\sum_{\varepsilon \in D_{k}}(-1)^{|\varepsilon|+k}\{[\alpha((\varepsilon, 0)), \alpha((\varepsilon, 1))]+[\alpha((\varepsilon, 0,0)), \alpha((\varepsilon, 0,1))]+[\alpha((\varepsilon, 1,0)), \alpha((\varepsilon, 1,1))]\}
\end{gathered}
$$


from Lemma 5

$$
\begin{aligned}
\left|c_{k}-c_{k+1}\right| & \leq 2 \cdot \tau_{k+1} \sum_{\varepsilon \in D_{k}}\left\{\alpha((\varepsilon, 0))^{2}+\alpha((\varepsilon, 1))^{2}\right\} \\
& \leq 2 \tau_{k+1} \sum_{\varepsilon \in D_{k}}\{\alpha((\varepsilon, 0))+\alpha((\varepsilon, 1))\}^{2} \\
& \leq 2 \tau_{k+1} \sum_{\varepsilon \in D_{k}} \alpha(\varepsilon)^{2}
\end{aligned}
$$

the last inequality B obtained from $0 \leq f_{0}(a, b, \theta)+f_{1}(a, b, \theta) \leq a$ but Since

$$
\begin{aligned}
\sum_{\varepsilon \in D_{k}} \alpha(\varepsilon)^{2} & =\sum_{\lambda \in D_{k-1}}\left\{\alpha((\lambda, 0))^{2}+\alpha((\lambda, 1))^{2}\right\} \\
\left|c_{k}-c_{k+1}\right| & \leq 2 \cdot \tau_{k+1} \sum_{\lambda \in D_{k-1}}\{\alpha((\lambda, 0))+\alpha((\lambda, 1))\}^{2}
\end{aligned}
$$

As a result

$$
\left|c_{k}-c_{k+1}\right| \leq 2 \cdot \tau_{k+1}\left(\alpha(0)^{2}+\alpha(1)^{2}\right)=2 \tau_{k+1}\left(a^{2}+b^{2}\right)
$$

is obtained [3].

Theorem 8. Every Archimedean almost f-algebra is commutative.

Proof. Let $a, \mathrm{~b} \in A^{+}$and $\theta \geq 0$. And let $\sum_{k=1} \tau_{k}<(1 / 2) \theta$ be the sequence of definite positive reel numbers. Take $\left\{\tau_{k}\right\}_{k=1}^{\infty}$, for $\alpha(\varepsilon)$ and $\varepsilon \in D_{k}(k=1,2, \ldots)$, $c_{k}$ is defined as in Definiton 13 and $c_{0}=[a, b]$.From Lemma 7

$$
\begin{aligned}
\left|c_{k}-c_{k+1}\right| \leq 2 \cdot \tau_{k+1}\left(a^{2}+b^{2}\right) \\
\left|[a, b]-c_{k}\right|=\left|\sum_{r=1}^{k}\left(c_{r-1}-c_{r}\right)\right| \leq \sum_{r=1}^{k}\left|\left(c_{r-1}-c_{r}\right)\right| \\
\leq \sum_{r=1}^{k} 2 \tau_{r}\left(a^{2}+b^{2}\right) \leq \theta\left(a^{2}+b^{2}\right)
\end{aligned}
$$

Besides since

$$
\begin{aligned}
c_{k} & =\sum_{\varepsilon \in D_{k}} \mp[\alpha((\varepsilon, 0)), \alpha((\varepsilon, 1))] \\
\left|c_{k}\right| & \leq \sum_{\varepsilon \in D_{k}}|[\alpha((\varepsilon, 0)), \alpha((\varepsilon, 1))]| \leq \sum_{\varepsilon \in D_{k}}\{|\alpha((\varepsilon, 0)) \alpha((\varepsilon, 1))+\alpha((\varepsilon, 1)) \alpha((\varepsilon, 0))|\}
\end{aligned}
$$

is obtained. From Lemma 6 (iii) and (ii)

$$
\sum_{\varepsilon \in D_{k}} \alpha((\varepsilon, 1))=\bigcup_{\varepsilon \in D_{k}} \alpha((\varepsilon, 1)) \leq \alpha(0)+\alpha(1)=a+b
$$


So

$$
\begin{gathered}
\left|c_{k}\right| \leq\left(\frac{1}{k+1}\right)\{\alpha(0)+\alpha(1)\} \sum_{\varepsilon \in D_{k}} \alpha((\varepsilon, 1)) \\
+\sum_{\varepsilon \in D_{k}} \alpha((\varepsilon, 1))+(\alpha(0)+\alpha(1)) \leq 2(a+b)^{2} /(k+1)
\end{gathered}
$$

for $\mathrm{k}=1,2, \ldots$ combining 2.6 and 2.7

$$
|[a, b]| \leq \theta\left(a^{2}+b^{2}\right)+2(a+b)^{2} /(k+1)
$$

is obtained. Since A is an Archimedean Riesz space, $|[a, b]| \leq \theta\left(a^{2}+b^{2}\right)$ for $\forall \theta>0$. Using Archimedean property of $\mathrm{A}|[a, b]|=0$ is obtained, in other words $a b=b a$. This proves that $\mathrm{A}$ is commutative. Every non Archimedean almost f-algebras can not be commutative [3].

\section{REFERENCES}

[1] M.Basly, And A. Triki, FF -Algebras Archimediennes reticnles, (1988).

[2] M.Basly, C.B. Huijmans, and B. Pagter, DE and A.Triki, On Unital Archimedean Lattice-Ordered algebras. J. Fac. Sci. Tunis, (1988).

[3] S.J.Bernau, and C.B.Huijmans Almost f -algebras and d -algebras Math. Groc. Camb. Mil. Spc, (1990), 287-308.

[4] G.Birkhoff, Lattice Theory. 3rd Edition. Amer. Math. Soc. Colloq. PubI. No. 25 American Mathematical Society, (1967).

[5] G.Birkhoff and R.S.Pierce Lattice Ordered Rings. An Acad. Brasil. Science, 28 (1956), 41-69.

[6] C.B.Huijmans, and B. Pagter, DE., Ideal tbeory in f.-algebras. Trans. Amer. Math. Sac. 269 (1982) 225-245.

[7] W. A. J.Luxemburg, Some Aspects of the Theory of Riesz spaces. Univ. Arkansas Lecture Notes Math.Vol. 4 (1979) Fayetteville

[8] W. A. J.Luxemburg, And A.C.Zaanen, Riesz Spaces. Vol. 1. North -Holland, (1971).

[9] P.N.Meyer, Banach Lattices; Springer Verlag, London, (1991).

[10] B.DE..Pagter, F -Algebras and orthomorphisms. Plı. D. Thesis, University of Leiden, (1981).

[11] C.Quint, Zur Darstelling Van Banach verbantsalgebren. Ph. D. Thesis, Universitat Darmstadt, (1984).

[12] A.C.Zaanen, Riesz Spaces, Vol. 2: North -Holland, (1983).

\section{Received: May 6, 2006}

